\title{
Disaster-Resilient Mobile Network Architecture
}

\author{
Karl Andersson \\ Pervasive and Mobile Computing Laboratory \\ Luleå University of Technology \\ SE-931 87 Skellefteå, Sweden
}

\author{
Ved P. Kafle \\ National Institute of Information and Communications \\ Technology (NICT) \\ Tokyo, Japan
}

\begin{abstract}
Conventional cellular mobile networks are likely to get disturbed easily when a natural disaster (earthquake, tsunami, flooding, etc.) hits an area because of their centralized control and high-power requirements. To address this issue, we propose a new mobile network architecture having distributed controls and ad-hoc configurations. This architecture exploits the locally available resources such as frequency spectrum and lively nodes to reconfigure the network and provide emergency services. We present its design and use cases.
\end{abstract}

Keywords-Disaster-Resilient Network, Future Internet, Mobile Network

\section{INTRODUCTION}

Natural disasters like earthquakes, floods and tsunamis are occurring from time to time in many places around the globe. Recent examples include the earthquake on Haiti in 2010, the Japanese earthquake and tsunami in 2011, the Chilean earthquake in 2011 and the Hurricane Sandy in USA in 2012.

While the need for communication and other types of ICT services is very high after such events, the communication infrastructure is often damaged to large extents, making services unavailable or at least heavily congested. Therefore, a number of standardization activities and research efforts have been started quite recently in the area of disaster-resilient communication.

One example of newly started standardization activities is the ITU-T Focus Group on Disaster Relief Systems [1]. Meanwhile, 3GPP has also started work on LTE for critical communications [2] and been planning to launch Proximity Services (ProSE) and Group call on LTE enablers (GCSE_LTE) in 3GPP Release 12. One already existing solution in the area is the ETSI TETRA standard [3], which currently is being developed further.

The research community in Japan has recently started interesting work in the field [4][5][6][7][8][9][10]. It is building on previous work from similar projects around the world, such as one in Taiwan [11]. Also, a group of European researchers are actively working on aerial base stations with opportunistic links for unexpected and temporary events [12]. The common goal of all these efforts is to design network architectures and solutions that are resilient to disasters. Typically solutions include the usage of a variety of radio access technologies in combination, often in mesh topologies.

In this paper, we propose a new mobile network architecture having distributed controls and ad-hoc configurations. This architecture exploits the locally available resources such as frequency spectrum and lively nodes to reconfigure the network on demand and provide emergency services. We present its design and use cases.

Our paper is organized in the following way: Section II covers related work, while Section III presents the proposed mobile network architecture. Section IV discusses a few use cases, while Section V, finally, concludes the paper and indicates future work.

\section{RELATED WORK}

Sakano et al. [4] proposed a disaster-resilient network architecture using specially designed movable and deployable resource units (MDRUs) being a transportable container that accommodates modularized equipment for networking, information processing, and storage. The architecture comprises three layers: network facility, network, and platform layers. Each MDRU forms a wireless access network around it to reach customer premises equipment. Existing optical fibers can often be used for connecting the MDRU to nationwide networks. Furthermore, the MDRU allows to be remotely operated and maintained, thus reducing the number of engineers needed after deployment. The authors point out the speed of installing the network and launching ICT services in the disaster area as the most important advantage offered by their solution.

Ngo et al. [5] studied throughput in wireless mesh networks with the solutions and architecture described in [6] deployed. The scenario included an MDRU being in charge of an area within the radius of 500 meters divided into seven cells. Each cell had a gateway in its center connected to the MDRU with a high capacity $25 \mathrm{GHz}$ band link. Two types of experiments were simulated using QualNet 5.1: a simple network and a network with realistic system parameters. The authors conclude that their proposal can provide Internet access to a significantly large population of users. They also point out that by using multi-channel backbone connections the network throughput can be improved.

Adachi et al. [6] presented principles for space-time coded multi-AP cooperative diversity. The idea behind the paper is that a single frequency network is formed by the access points that have survived the disaster, where one of the access points takes on the role as gateway to the core network and signal processing center for space-time coding and decoding. Spacetime block coded joint transmit/receive diversity and simple Alamouti's space-time coded transmit diversity are mentioned to be used for downlink and uplink transmissions, respectively, allowing for an arbitrary number of antennas while maintaining 


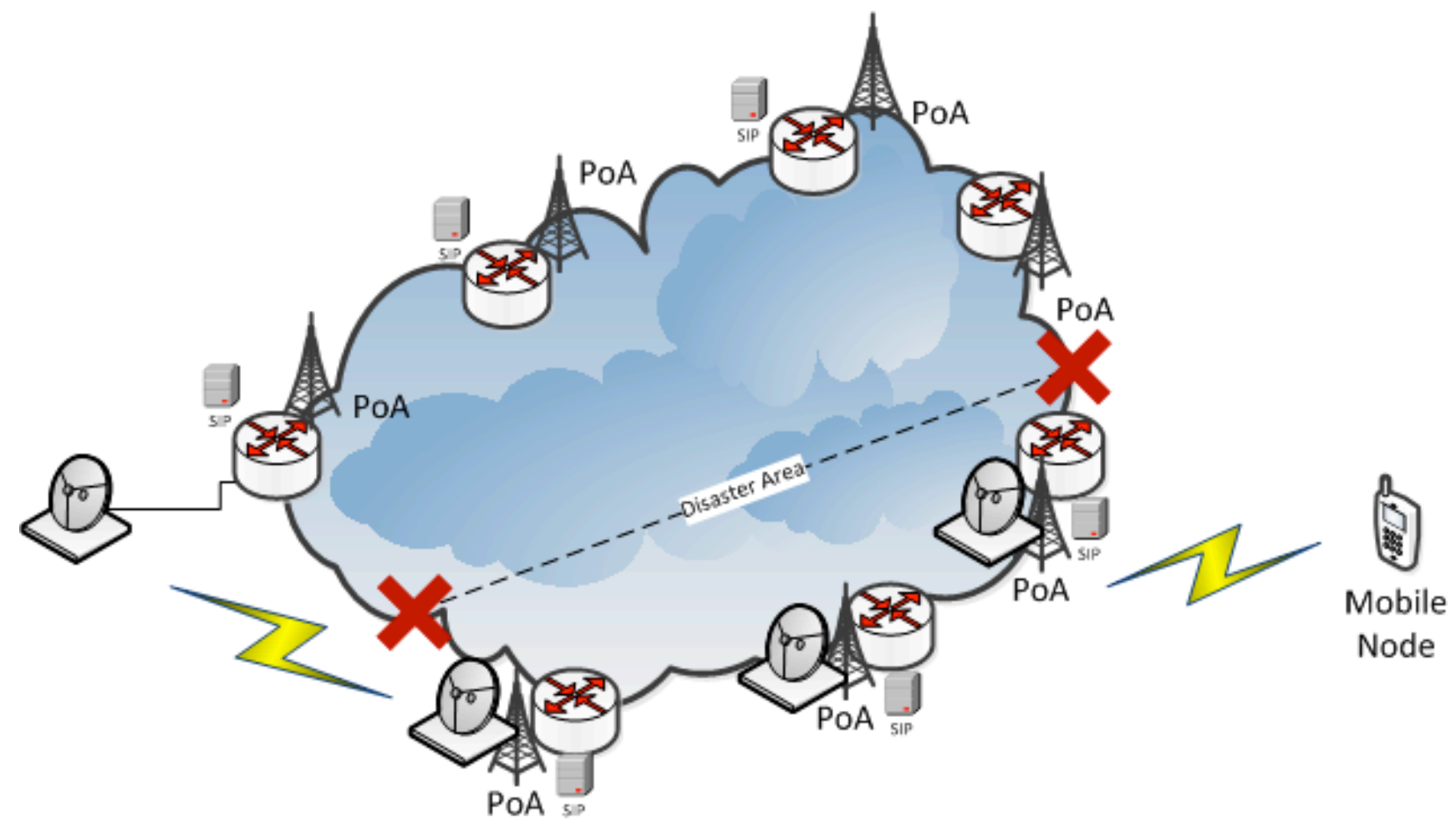

Figure 1. Proposed architecture.

the same coding rate. Simulations showed improvements both for $10 \%$ outage throughput and $90 \%$ outage throughput when using two access points compared to only using one access point.

Furthermore, Adachi et al. [7] proposed secured information service platforms for disaster applications. Two types of services were sketched: secured group communication and push-type personalized transmission.

In [8], Ohyama et al. described how vehicles could be used as network nodes in temporary disaster information networks. The basic observation behind the ideas presented is the fact of DSRC (Dedicated Short Range Communication) systems gradually coming into practical use in some countries. Both mobile roadside units (RSUs) and vehicular on-board units (OBUs) are used as network nodes in a multi-hop network, while end-users are using regular smart phones. The system is designed to use a variety of protocols for vehicular networking including ARIB STD-75 (operating at the $5.8 \mathrm{GHz}$ band), ARIB STD-T109 (operating at the $760 \mathrm{MHz}$ band), and IEEE $802.11 \mathrm{p}$ [13] (operating at the $5.8 \mathrm{GHz}$ band).

Moreover, Kishi et al. [9] described concepts, requirements, and challenges for the recovery of base stations' backhaul connectivity under disaster situations. The basic concept presented is to use neighbor regional wireless access networks connecting base stations to backhaul, possibly by using wireless LAN relay nodes to overcome long distances. Deployments of such relay nodes are discussed. Also, the authors point out that the connection between the base station and the aggregator must implement the PLMN-specific protocol normally used for that link.
Finally, Uchida et al. [10] proposed a Never Die Network (NDN) consisting of a cognitive wireless network and a satellite network so that the best possible wireless links and routes are selected. The optimal link selection adapts an extended Analytic Hierarchy Process (AHP) method by a change of network environment and user policy during a disaster. Provided the network environment or user environment can be changed, a proper route selection method is conducted by an extended Ad Hoc On-Demand Distance Vector (AODV) method with Min-Max AHP values.

All the above-mentioned related work deal with different aspects of disaster-resilient network architectures. This paper presents an overall design for such an environment building on our own previous work in related areas such as interworking techniques and architectures for heterogeneous wireless networks [14], a proposal on distributed mobility management [15], and a proposal on locator id separation for mobility management in the new generation network [16].

\section{Proposed Mobile Network ArChitecture}

The general goal for our work has been to increase disaster resilience in networks. Before going into the details of our proposal, we make some initial observations and identify needs and requirements.

\section{A. Initial Observations}

We build our architecture proposal based upon the following observations:

1) Many mobile phones and handsets today have multiple radio access technology capabilities built into them where 


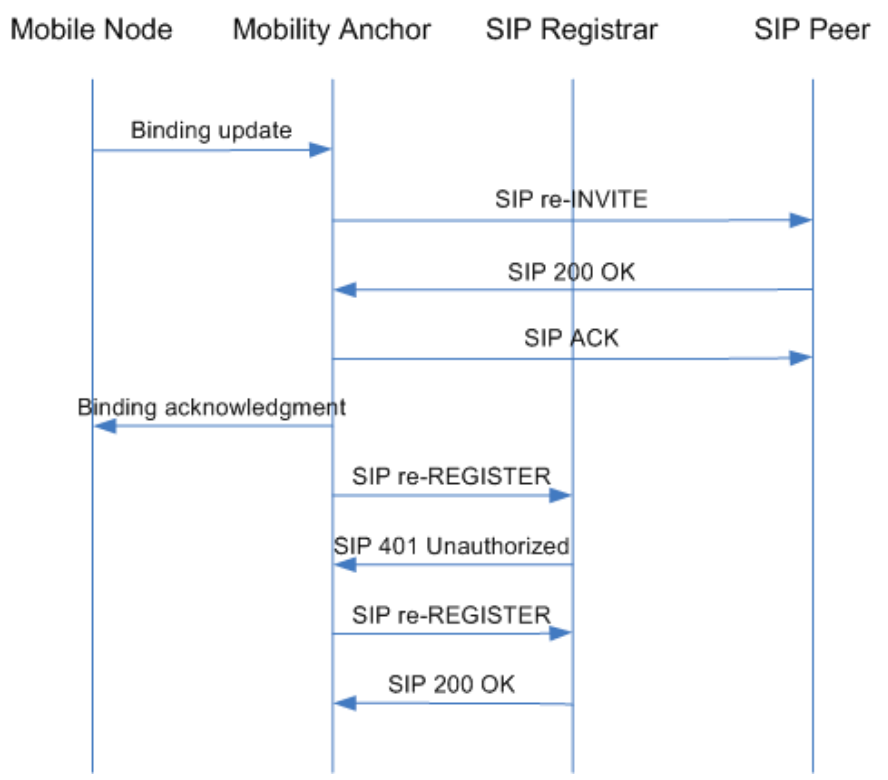

Figure 2. Signaling scheme.

Wi-Fi and LTE are the most commonly spread technologies around the globe;

2) Many mobile phones and handsets today are geolocation aware through GPS;

3) Mobility-related functionality is often handled in a centralized anchor point handling millions of subscribers in the backhaul network;

4) Base stations and access points that lost their backhaul connection can seldom continue to operate in an autonomous way;

5) Direct communication in a peer-to-peer manner (without the involvement of access points or base stations) is poorly supported; and

6) Multimedia and other types of content is delivered to end users using content delivery mechanisms.

Before describing the proposed solution, we are listing the key needs and requirements we are targeting.

\section{B. Needs and Requirements}

The key needs and requirements for the architecture are the following: mobility, reliability, robustness, and scalability.

For mobility, we argue that mobile features are needed for rescue staff workers, police forces, and any other public authority staff working in the disaster area, which may cover a large area.

For reliability and robustness, we argue that functions must be able to continue to operate in rough circumstances because of the type of applications we are targeting.

For scalability, we argue that solutions must scale with an increasing number of users. This is very much needed since many natural disasters occur in densely populated areas.

\section{Proposed Network Architecture}

Building upon the aforementioned observations as well as the identified needs and requirements, we build our proposal on a heterogeneous wireless network environment with 3GPP LTE and Wi-Fi radio access technologies. The core of our proposed solution is targeted towards the access routers in those two radio access network technologies, where we propose new functionality for increasing the disaster resiliency having them to operate in an autonomous manner. This includes both new functions in Wi-Fi access points and LTE eNodeB Point of Attachments (POAs) where they contain functions for proximity-based services and group communication without the need for access router being connected to any core network by their standard backhaul connection.

We suggest a flat network architecture where each base station and $\mathrm{Wi}-\mathrm{Fi}$ access point takes on routing functionality, often referred to as a flat IP architecture. Moreover, we propose introducing mobility anchors in these access routers to allow for distributed and autonomous mobility management. For mobility handling, in our proposal, mobile nodes will have the ability to set up sessions with a) full mobility, b) notified mobility, or c) no mobility. Sessions started with the full mobility option chosen will be mobility managed using Mobile IPv6 and HIMALIS [16] in a decentralized manner, so that the access router having started the session maintain location management and connection migration functions for that particular flow. For sessions started with the notified mobility option the access router having started the session will notify the mobile node of the change of point of attachment. Other sessions will not be mobility handled at all.

The mobility functionality provided in access routers is proposed to come along with SIP registrars where signaling 

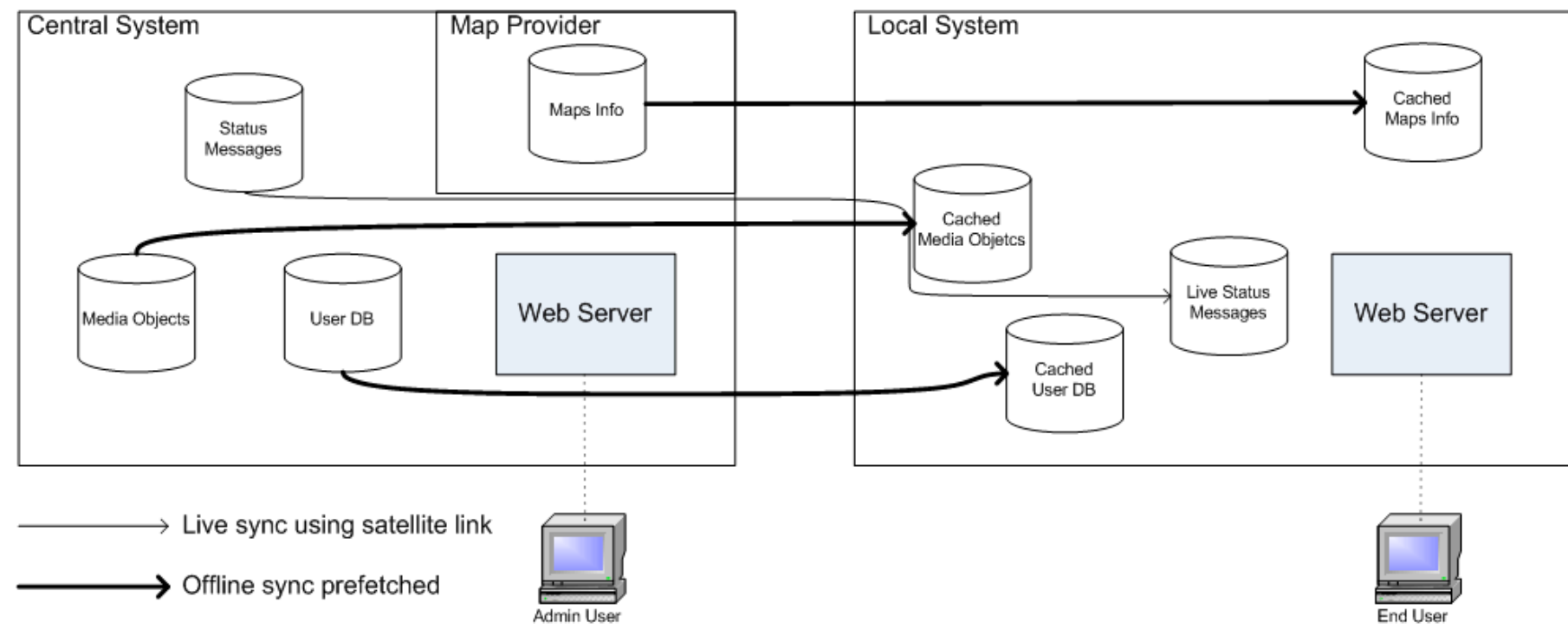

Figure 3. P roposed solution for application information sharing.

for notified mobility type of sessions is handled by using the SIP protocol [17]. A satellite backhaul link from the access router will send location update messages to a central SIP registrar. A hierarchy is built up, so that local mobility management within the local autonomous domain will not be visible to the central SIP registrar.

For the case of HIMALIS-based mobility management scheme, the ID/locator split concept is applied to achieve seamless mobility across networks of heterogeneous protocols (e.g. IPv4 and IPv6).

The proposed architecture solution is depicted in Figure 1, while the proposed signaling scheme is depicted in Figure 2.

\section{Proposed Status Updating Application}

Our proposal also contains a status updating application being designed like an in-flight entertainment system where most material is stored in a server nearby the user where there is no need for backhaul connectivity. By adding functionality for updating certain information elements dynamically from some locations in the backhaul network using low bandwidth satellite links, government agencies can convey mission critical information also to people in disaster areas. This part of the proposal is depicted in Figure 3.

\section{E. Mobile Ad-hoc Networking Functionality}

The next part of our proposal deals with mobile ad-hoc networking functionality. The idea is that users normally covered by access points or base stations that stopped to operate after a disaster should be able to communicate using intermediate nodes connected to access points and base stations actually working. For this purpose smart phones capable of sharing Internet connections are used in combination with mesh topologies. Typically the smart phone sharing its Internet connection over Wi-Fi acts as a mesh portal. This part of the proposal is depicted in Figure 4.

\section{F. Network Maps and Node Maps}

Finally, we also propose network maps and node maps for information sharing purposes to be maintained using the location-awareness capabilities of modern smartphones. The map information itself will be stored in access routers, so that there is no need for connection to the backhaul network for this part of the solution. The networks and node maps solution is a live counterpart to "Wireless Geographic Logging Engine" [18]. Figure 5 depicts that part of our proposed solution.

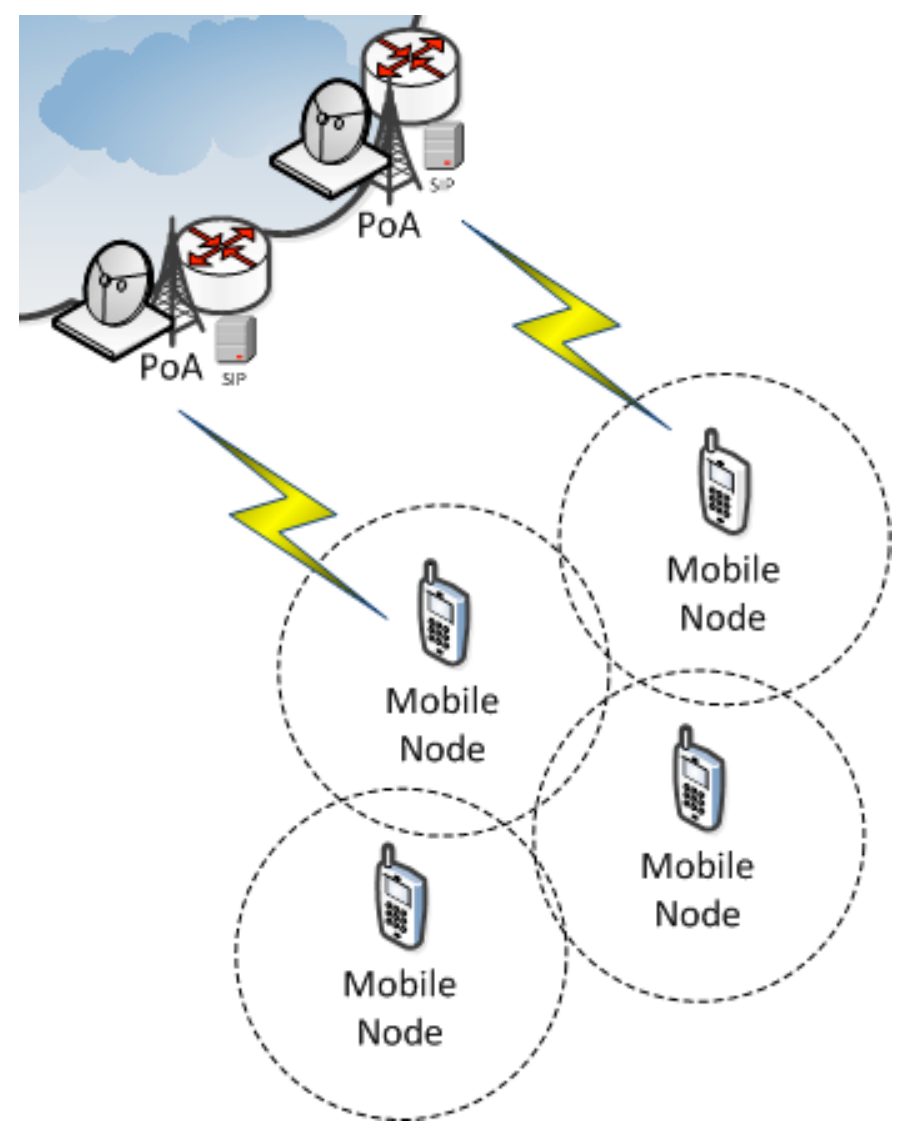

Figure 4. Proposed mobile ad-hoc networking architecture. 


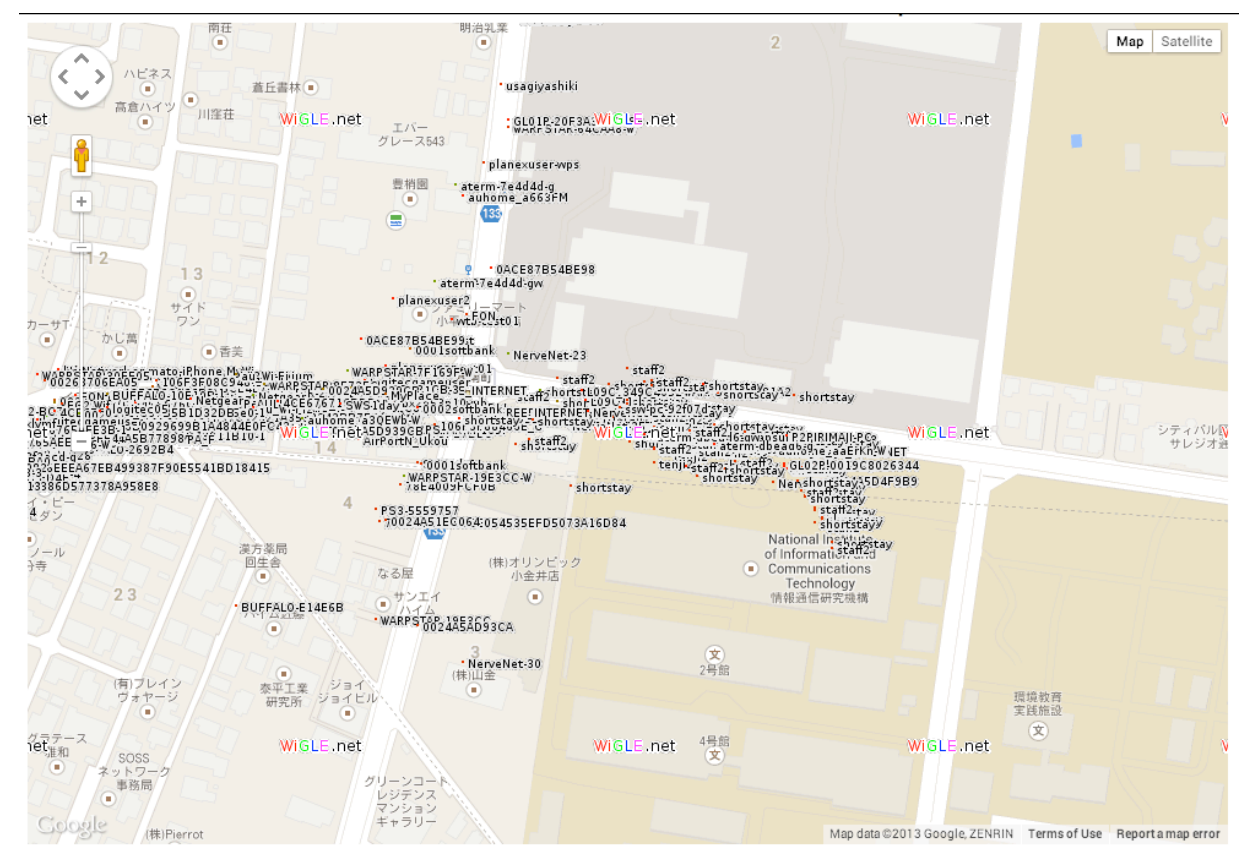

Figure 5. Proposed solution for network maps and node maps, example from wigle.net [18].

\section{USE CASES}

For evaluation of the proposed network architecture, we identified a set of use cases, namely:

1) Firefighters and rescue staff using communication hotspots;

2) Family members and dependents having needs to communicate with each others; and

3) Government agencies "pushing" information to disaster areas.

For the first use case our proposed architecture will support firefighters and rescue staff in a way they can back off to hotspots not totally destroyed and communicate. Also, using the ad hoc features they can communicate while in heavily affected areas.

For the second use case, the SIP registrars deployed along with access routers can convey information about family members in a very distributed fashion.

For the third use case, government agencies can push information also to disaster areas using satellite low bandwidth links for distribution of mission critical short message type of information. The information will be accessible from local web servers located together with access points using an inflight entertainment system like setting.

\section{CONCLUSION AND FUTURE WORK}

As discussed in the previous section, our proposal comes handy when dealing with firefighters and rescue staff, family members communication, and government agencies having a need to convey mission critical information.

There are, however, a set of questions that need further attention including handling of multiple interfaces in handsets, standardization of distributed and decentralized mobility management, and offloading to Wi-Fi from LTE networks.

For this purpose, we will closely follow standardization work in IETF (studying multiple interfaces in handsets in MIF WG [19] as well as distributed and decentralized mobility management in DMM WG [20]) and 3GPP (standardizing WiFi offloading techniques in LTE networks [21]). Moreover, HIMALIS is also trying to propose a solution for DMM.

For future work, we intend to perform a comparative study benchmarking different architectural settings for mobility management: conventional Mobile IPv6 operating form the IP layer, SIP operating from the application layer, and HIMALIS operating from the identity layer, which is a new layer inserted between the transport and network layers.

Moreover, we intend to explore security aspects further.

\section{ACKNOWLEDGMENT}

This work was supported by Japan Society for the Promotion of Science (JSPS) under grant 13545.

\section{REFERENCES}

[1] ITU-T Focus Group on Disaster Relief Systems (FG-DR\&NRR), Network Resiliency and Recover, http://www.itu.int/en/ITUT/focusgroups/drnrr

[2] 3GPP, LTE for Critical Communications, http://www.3gpp.org/LTE-forCritical-Communications

[3] ETSI, TErrestrial Trunked RAdio (TETRA), http://www.etsi.org/technologies-clusters/technologies/tetra

[4] T. Sakano, Z. Md. Fadlullah, T. Ngo, H. Nishiyama, M. Nakazawa, F. Adachi, N. Kato, A. Takahara, T. Kumagai, H. Kasahara, and S. Kurihara, "Disaster-Resilient Networking: A New Vision Based on Movable and Deployable Resource Units," IEEE Network Magazine, vol. 27, no. 4, pp. 40-46, July-August 2013.

[5] T. Ngo, H. Nishiyama, N. Kato, Y. Shimizu, K. Mizuno, and T. Kumagai, "On the Throughput Evaluation of Wireless Mesh Network 
Deployed in Disaster Areas," in Proceedings of 2013 International Conference on Computing, Networking and Communications, Wireless Communications Symposium, 30-31 July 2013, pp. 413-417.

[6] F. Adachi and S. Kumagai, "Multi-AP Cooperative Diversity for Disaster-resilient Wireless LAN," in Proceedings of 2012 15th International Symposium on Wireless Personal Multimedia Communications (WPMC), 24-27 Sept. 2012, pp. 27-28.

[7] F. Adachi, K. Tokuda, and S. Ohmori, "Secured information service platforms effective in case of disasters - Using multilayered communications network," in Proceedings of 2012 15th International Symposium on Wireless Personal Multimedia Communications (WPMC), 24-27 Sept. 2012, pp. 348-349.

[8] T. Ohyama, Y. Kaneko, K. Asano, and M. Hamaguchi, "Multi-hop Communication and Multi-protocol Gateway by Using Plural ITS," in Proceedings of 2012 15th International Symposium on Wireless Personal Multimedia Communications (WPMC), 24-27 Sept. 2012, pp. 217-220.

[9] Y. Kishi, N. Kurishima, Y. Fujimoto, Y. Ikenoue, H. Iwama, H Uchiyama, M. Matsuda, M. Shimizu, K. Fukushima, and Y. Hirata, "Rapid Recovery of Base Station Backhaul by the Neighbor Regional Wireless Access Network and Its Extension with the Multi-hop Wireless LAN Relay," in Proceedings of 2012 15th International Symposium on Wireless Personal Multimedia Communications (WPMC), 24-27 Sept. 2012, pp. 221-224.

[10] N. Uchida, K. Takahata, Y. Shibata, and N. Shiratori, "Never Die Network Based on Cognitive Wireless Network and Satellite System for Large Scale Disaster," in Journal of Wireless Mobile Network, Ubiquitous Computing, and Dependable Applications 3(3):74-93, September 2012.
[11] Y.-T. Chiu, "Japan Looks to Taiwan to Disaster-Proof Telecom," IEEE Spectrum, February 2013

[12] European Commission. FP7 ABSOLUTE, Aerial Base Stations with Opportunistic Links for Unexpected \& Temporary Events, http://www.absolute-project.eu/

[13] IEEE 802.11p-2010. Wireless LAN Medium Access Control (MAC) and Physical Layer (PHY) Specifications Amendment 6: Wireless Access in Vehicular Environments, July 2010.

[14] K. Andersson, "Interworking Techniques and Architectures for Heterogeneous Wireless Networks," in Journal of Internet Services and Information Security 2(1/2):22-48, February 2012.

[15] K. Andersson and M. Elkotob, "Rethinking IP Mobility Management," in Journal of Wireless Mobile Networks, Ubiquitous Computing, and Dependable Applications 3(3):41-49, September 2012.

[16] V. P. Kafle and M. Inoue, "Locator ID separation for mobility management in the new generation network [Invited paper]," Journal of Wireless Mobile Network, Ubiquitous Computing, and Dependable Applications, 1(2/3):3-15, October 2010.

[17] H. Schuklzrinbne and E. Wedlund, "Application-layer moblity using SIP," in ACM SIGMOBILE Mobile Computing and Communications 4(3):47-57, July 2000.

[18] Wireless Geographic Logging Engine, http://wigle.net

[19] IETF Working Group on Multiple Interfaces, https://tools.ietf.org/wg/mif

[20] IETF Working Group on Distributed Mobility Management, https://tools.ietf.org/wg/dmm

[21] 3GPP TS 23.261. IP flow mobility and seamless Wireless Local Area Network (WLAN) offload, September 2012. 